\title{
ECONOMÍA DEL TURISMO Y ETNICIDAD EN RAPA NUI
}

\author{
TOURISM ECONOMICS AND ETHNICITY IN RAPA NUI
}

\author{
Hans Gundermann* y Roberto Rojas **
}

Tratamos aquí la industria del turismo y su relación con los cambios demográficos, la distribución ocupacional, la estratificación socioeconómica y la etnicidad en Rapa Nui. Sostenemos que la economía de Rapa Nui y la etnicidad están estrechamente relacionados, y que todo ello arrastra consecuencias en la producción de diferenciación económica y desigualdades sociales internas. Se consignan varios hitos. Primero, el desarrollo de infraestructura urbana y servicios, transporte aéreo, cabotaje y comunicaciones, dependiente directamente de inversiones públicas que posibilitó la actividad turística. Segundo, el fomento de la industria del turismo donde al Estado, unido a un muy activo interés rapanui, cupo un rol impulsor decisivo. Se presentan cambios demográficos, considerando una perspectiva temporal amplia, destacando el rápido crecimiento de la población en la isla, prestando atención al considerable flujo de continentales que se radican, encontrando empleos en los servicios del Estado, las inversiones públicas y, sobre todo, en el desarrollo de instalaciones y servicios para el turismo. En el empleo público y en el turismo los rapanui han llegado a tener una sustancial presencia, unido a una considerable influencia, en un caso, y control, en el otro, de estas dos principales fuentes de ingresos de la isla. Los rapanui mantienen una posición de predominio en la economía del turismo y en la propiedad de gran parte de las empresas y actividades independientes asociadas a ella, mientras los continentales, radicados o con estadías temporales, tienen una posición, en general, muy numerosa pero subordinada a empleos asalariados y emprendimientos por cuenta propia menores. Por lo demás, es una economía en que predominan unidades familiares, especialmente en el rubro de alojamientos, con una ocupación mayoritariamente femenina.

Palabras claves: Industria del turismo, etnicidad, cambios demográficos, distribución ocupacional, estratificación socioeconómica.

We discuss here the tourism industry and its relationship with demographic changes, occupational distribution, socio-economic stratification and ethnicity in Rapa Nui. We maintain that the Rapa Nui economy and ethnicity are closely related, and that all this has consequences in the production of economic differentiation and internal social inequalities. Several milestones are recorded. First, the development of urban infrastructure and services, air transport, cabotage and communications, directly dependent on public investments that made tourism possible. Second, the promotion of the tourism industry where the State, together with a very active Rapanui interest, played a decisive driving role. Demographic changes are presented, considering a broad temporal perspective, highlighting the rapid growth of the island's population, paying attention to the considerable flow of continentals that settle, finding jobs in State services, public investments and, above all, in the development of facilities and services for tourism. In public employment and tourism, the Rapanui have come to have a substantial presence, coupled with considerable influence, in one case, and control, in the other, of these two main sources of income on the island. The Rapanui maintain a dominant position in the tourism economy and in the ownership of a large part of the independent companies and activities associated with it, while the continental ones, settled or with temporary stays, have a position, in general, very numerous but subordinate to salaried jobs and minor self-employment ventures. For the rest, it is an economy dominated by family units, especially in the housing sector, with a predominantly female occupation.

Key words: Tourism industry, ethnicity, demographic changes, occupational distribution, socioeconomic stratification.

\section{Introducción}

La modernización, sus efectos políticos y económicos, los cambios demográficos, las relaciones interétnicas y la economía del turismo en Rapa Nui están relacionados. La expansión de la industria del turismo ha producido un sostenido crecimiento de la población residente en la isla. Los rapanui, continentales (“conti” en el lenguaje local) y extranjeros son las categorías de población que habita, trabaja y son parte de la sociedad isleña. Al respecto, se advierte diferenciación ocupacional de continentales y extranjeros respecto de la población rapanui. Estos habrían realizado una incorporación activa al proceso de modernización, ganando presencia notoria en la administración local del Estado

\footnotetext{
* Instituto de Investigaciones Arqueológicas y Antropológicas (IAA), Universidad Católica del Norte. San Pedro de Atacama, Chile. Correo electrónico: hgunder@ucn.cl

** Programa de Doctorado (UCN-UTA), Instituto de Investigaciones Arqueológicas y Antropológicas (IAA), Universidad Católica del Norte. San Pedro de Atacama, Chile. Correo electrónico: robertorojaspantoja@gmail.com
} 
$\mathrm{y}$, sobre todo, en la industria del turismo. Son ellos los controladores (propietarios, administradores) de la gran mayoría de los emprendimientos familiares y empresas asociadas al rubro turístico.

Un conjunto de factores sociohistóricos como la "Ley Pascua" No 16.441 de 1966 y sus normas especiales, la creación de infraestructura urbana, portuaria, de transporte aéreo y comunicaciones, el significativo fomento económico estatal y una población mayoritariamente rapanui en la isla, habría otorgado ventajas iniciales para el gran interés e iniciativa de personas y familias locales en este campo. Desde el posicionamiento logrado y, más tarde, con la afluencia de trabajadores continentales, habrían logrado prevalencia y dominio sobre el turismo. Por consiguiente, además de mantenerse en el empleo público, los continentales se insertan en la economía del turismo como trabajadores y empleados de los negocios turísticos rapanui y, en esa medida, mantendrían una posición laboral y de ingresos subordinada. Tienen también presencia en actividades por cuenta propia de todo tipo. Por tratarse principalmente de trabajadores, prestadores de servicios y cuenta propia menores (emprendimientos familiares pequeños, aunque también algunos casos de miniempresas), tenderían a ocupar posiciones en los estratos bajos de la estructura económica del turismo y social de la isla.

Se intenta en lo que sigue entregar información y argumentos que sustenten la línea de interpretación aquí presentada, enfatizando modernización, desarrollo de la industria del turismo, cambios demográficos, participación y posicionamiento económico rapanui y continental en ella. Otorgamos visibilidad a factores concurrentes históricos y económicos que consideramos de importancia, sin pretender agotar las determinaciones posibles.

\section{Acción desarrollista y crecimiento del turismo}

Desde la segunda mitad de la década de 1960 y con la implementación de la Ley Pascua $\left(\mathrm{N}^{\circ} 16.441\right)$, la acción desarrollista del Estado se realizó tanto en la creación de infraestructura como en la habilitación de servicios. Consideró distintos rubros y, como era de esperarse, se ejecutó progresivamente. Los más importantes fueron portuario y de cabotaje, el aéreo y de comunicaciones, la urbanización y construcción de viviendas y, por último, los servicios básicos y sociales. El conjunto profundizó la conexión con el continente y proveyó una mejoría considerable a la isla, si se compara con las condiciones materiales de la vida local previa. Como resultado de este proceso se establecen las bases para el desarrollo del turismo, que adquiere progresivamente la condición de principal economía de la isla. Todo ello incidió en la calidad de vida de los isleños y en la posibilidad concreta de poder recibir y brindar servicios turísticos (con otros detalles y alcances también en Rojas y Gundermann, 2020).

El turismo es una actividad planificada, por una o más personas, para explorar o pasar un tiempo prolongado realizando actividades no productivas, de recreación u ocio, en un lugar distinto al que residen ${ }^{1}$. El interés de visitar por curiosidad e interés no económico Rapa Nui tiene diversos antecedentes. Mencionemos uno al que se le reconoce indudable importancia. La expedición de Thor Heyerdahl en 1955-56 consideró en sus preparativos y ejecución una intensa labor de propaganda y difusión, lo que como consecuencia estimuló el interés turístico y cambió de manera posiblemente irreversible la imagen global de Rapa Nui (Porteous, 1981; Fischer, 2005; Muñoz, 2018; Foerster y Montecino, 2018). También contribuyó lo suyo la temprana identificación de sitios y monumentalidad arqueológica de la isla y su interés intelectual, científico, museológico, al igual que su exotismo. De esta manera, el turismo ha estado vinculado intrínsecamente a la identificación de Rapa Nui como un destino remoto con una historia humana muy especial, de la que dan testimonio la arqueología de los sitios Orongo, Rano Raraku y los ahu de Vinapu. Son los sitios que presentan la mayor atracción para los turistas, pero no la única. Más recientemente, su definición nacional e internacional como un destino turístico especial y la promoción comercial a ello asociada contribuyen a la notoriedad que acompaña su mención. Una reciente película, una telenovela nacional y el festival cultural polinésico anual Tapati Rapa Nui hacen su aporte.

Desde 1967 los vuelos regulares posibilitaron el traslado regular de personas a Rapa Nui. Ese año viajaron 444 personas, número que aumentó a 11.403 en 1971. La oferta de la Línea Aérea Nacional (LAN) era de dos vuelos a la semana, pensada para un plan de turismo de 3 a 4 días. En 1970 el hotel Honsa (perteneciente a Hotelera Nacional, una empresa estatal), con dos años de funcionamiento, ofrecía 120 camas y una capacidad extra para 20 personas en un campamento del mismo 
hotel. Las casas de los isleños estaban dentro de la oferta de alojamiento. En ese momento se ofrecían 100 camas, casi la mitad de la oferta total de la isla. Al respecto, los problemas habitacionales $y$ de hospedajes fueron resueltos no solo con la construcción de viviendas por parte del Estado sino con una intervención de la comunidad, que construyó un mundo de hospitalidad para los "pasajeros" acorde a sus expectativas, transformando sus viviendas en "residenciales" (Foerster y Montecino, 2018: 236-237). Ya en 1975 se crea la primera agencia de turismo llamada "Te Mahina", que contaba con 9 vehículos motorizados para las visitas al interior de la isla y 6 particulares para transporte turístico. El mismo año se formaliza Martin Travel y Anakena Tours. El año siguiente Manutara Tours, Aku Aku Tours, Iorana Tours, y Archeological Travel Service (SERNATUR, 1979).

El flujo de personas que con fines turísticos visitan Rapa Nui presenta un crecimiento sostenido desde finales de la década de 1960 hasta el presente. Los principales consumidores de turismo son hoy de nacionalidad chilena, cercano a 50\% del total de visitantes. La otra mitad comprende un amplio segmento de población proveniente de América del Norte, el continente europeo y asiático (cf. más abajo
Tabla 1). Para el 2002 se tiene un registro oficial de 17.305 visitantes, que aumenta hasta 58.459 en el $2013^{2}$. Desde la década del 2000 en adelante los pasajeros de nacionalidad chilena continúan representando la mitad de la demanda turística, seguido por el flujo de turistas de Francia, EE.UU., Japón, España, Alemania e Inglaterra (véase Tabla 1).

Las estimaciones de visitantes que se manejan para el 2014 hasta el 2016 parecen imprecisas, por ausencia de conteos oficiales sistemáticos confiables. Para el 2017 y 2018 tenemos información de Ma'u Henua, la comunidad polinésica rapanui que administra el Parque Nacional Rapa Nui. Atendiendo al número de boletos vendidos para visitar los sitios culturales y de paisaje de la isla, en su mayoría dentro del mencionado parque, la cifra de visitantes entre enero y diciembre de 2018 sería de 149.915 (un boleto permite a una persona visitar todos los sitios que le interesen $)^{3}$. Entre el 2013 y 2018 la afluencia de turistas entonces se triplicó. Aunque se verifica un explosivo aumento en el número de visitantes, tal crecimiento no se explica solo por causa de un aumento del interés en dirigirse a esta isla; creemos que también concurre a resaltar la diferencia el conteo insuficiente en los años que se realiza la comparación $n^{4}$.

Tabla 1. Flujo de turistas por nacionalidad (2002-2013)

\begin{tabular}{lrrrrrrrrrrr}
\hline País/Año & \multicolumn{1}{c}{2002} & \multicolumn{1}{c}{2003} & \multicolumn{1}{c}{2004} & \multicolumn{1}{c}{2005} & 2006 & 2007 & 2008 & 2009 & \multicolumn{1}{c}{2010} & 2011 & 2013 \\
\hline Chile & 3.720 & 3.571 & 6.473 & 6.889 & 8.621 & 7.974 & 11.260 & 13.358 & 13.425 & 12.963 & 30.365 \\
Francia & 3.201 & 3.257 & 4.038 & 4.291 & 3.790 & 4.079 & 3.252 & 5.121 & 3.328 & 3.272 & 3.281 \\
EE.UU. & 2.128 & 1.945 & 2.507 & 2.313 & 2.846 & 4.061 & 4.275 & 2.390 & 2.630 & 2.671 & 4.479 \\
Japón & 1.661 & 1.599 & 2.116 & 2.075 & 2.087 & 2.431 & 2.785 & 2.390 & 1.970 & 1.197 & 2.318 \\
Alemania & 986 & 1.127 & 1.437 & 2.037 & 1.990 & 1.749 & 1.712 & 2.693 & 1.763 & 1.555 & 1.666 \\
Inglaterra & 969 & 1.015 & 1.517 & 1.475 & 1.585 & 1.618 & 1.423 & 1.285 & 1.025 & 1.053 & 1.017 \\
Australia & 249 & 624 & 926 & 811 & 1.201 & 880 & 954 & - & - & 825 & - \\
Italia & 605 & 846 & 1.027 & 1.130 & 1.283 & 1.168 & 936 & 1.219 & 809 & 819 & 1.251 \\
España & 536 & 761 & 1.052 & 1.255 & 1.684 & 1.654 & 1.664 & 1.524 & 1.065 & 893 & 1.485 \\
Brasil & 346 & 219 & - & 478 & 708 & 991 & 1.122 & 918 & 814 & 876 & 2.619 \\
N. Zelanda & 311 & 297 & - & 597 & - & - & - & - & - & - & - \\
Suiza & - & 438 & 471 & 697 & - & - & - & - & - & - & - \\
Canadá & - & - & - & 407 & - & - & - & - & - & - & - \\
Argentina & - & - & - & 318 & - & - & - & - & 816 & 895 & - \\
China & - & - & & - & - & - & - & 852 & - & - & - \\
\hline Total & 17.305 & 18.601 & 24.175 & 28.348 & 32.497 & 33.889 & 46.823 & 43.869 & 60.259 & 59.089 & 58.459 \\
\hline
\end{tabular}

Fuente: Elaboración propia a base del Informe Anual de Turismo que publica el Instituto Nacional de Estadísticas (INE) y Servicio Nacional de Turismo (SERNATUR), 2002-2013. 


\section{Cambios demográficos}

Previo al proceso de modernización iniciado en la década de 1960, se tiene en 1952 una población residente de 800 personas, en su gran mayoría rapanui (Porteous, 1981:220). En 1958 asciende a 995 y para 1970 llega a 1405 (McCall, 1975:469). Este último crecimiento se asocia con el "desembarco del Estado" y tuvo un efecto progresivo en la construcción de casas e instalación de servicios (Porteous, 1981: 220). Cf. Tabla 2.

Más adelante, entre 1982 y 2017, se verifica un crecimiento que cuadruplica la población total residente. En efecto, tomando como base los 1936 residentes de 1982 según el censo nacional de ese momento, durante los 35 años transcurridos hasta el último censo la población local prácticamente se cuadruplicó (llegó en 2017 a 7750 individuos) y ello no es explicable solo por el crecimiento interno o vegetativo. La mejor explicación se encuentra en el poder de atracción que ejerce la expansión de la economía del turismo en Rapa Nui, lo que genera una muy activa inmigración. La oscilante proporción de personas rapanui se entiende por el registro censal como adscripción; esto es, una inscripción subjetiva no basada en atributos objetivos (lengua por ejemplo). En 1992 la autoadscripción rapanui no tenía la extensión actual. Por tanto, el aumento en 2002 de la población rapanui (de 42,5\% en 1992 a $61,3 \%$ en 2002) no obedece, según los antecedentes disponibles, a un crecimiento interno o migración de retorno, sino a un desarrollo de la adscripción étnica. Manteniendo esa alta adscripción en el censo de 2017 el retroceso en la proporción de rapanui respecto de la población total obedecería a un aumento de la inmigración continental, y extranjera en menor medida. El crecimiento de población continental en la isla corresponde con el período, posterior a 2000, de más rápido crecimiento de los visitantes con fines de turismo y con la ampliación de la oferta turística local de productos y servicios. Cf. Tabla 3.

Durante el período 1982-2017 destaca el aumento notorio, en términos relativos, de la población

Tabla 2. Población rapanui y continental entre1958 y 1972

\begin{tabular}{lccccc}
\hline Año & Total & $\begin{array}{c}\text { Total, hombres } \\
\text { continentales y } \\
\text { extranjeros }\end{array}$ & $\begin{array}{c}\text { Total, mujeres } \\
\text { continentales y } \\
\text { extranjeras }\end{array}$ & $\begin{array}{c}\text { Total, continentales y } \\
\text { extranjeros }\end{array}$ & $\begin{array}{c}\% \text { de población } \\
\text { continental y } \\
\text { extranjera }\end{array}$ \\
\hline 1958 & 938 & 57 & 58 & 115 & 11 \\
1959 & 968 & 59 & 54 & 113 & 10 \\
1960 & 1.035 & 70 & 55 & 125 & 11 \\
1961 & 1.065 & 55 & 65 & 120 & 10 \\
1963 & 1.010 & 74 & 46 & 120 & 11 \\
1965 & 974 & 90 & 82 & 172 & 31 \\
1970 & 1.108 & 297 & 253 & 491 & 27 \\
1972 & 1.355 & 259 & 511 & \\
\hline
\end{tabular}

Fuente: McCall, 1975:469.

Tabla 3. Población residente en Rapa Nui y su crecimiento (1982-2017)

\begin{tabular}{lcccc}
\hline \multicolumn{1}{c}{ Año } & 1982 & 1992 & 2002 & 2017 \\
\hline Rapanui & - & $1.175(42,5 \%)$ & $2.324(61,3 \%)$ & $3.408(44,0 \%)$ \\
No rapanui & - & $1.589(57,5 \%)$ & $1.467(38,7 \%)$ & $4.342(56,0 \%)$ \\
\hline Total población & $1.936(100 \%)$ & $2.764(100 \%)$ & $3.791(100 \%)$ & $7.750(100 \%)$ \\
\hline
\end{tabular}

Fuente: Elaboración propia a base de los datos del Censo de Población y Vivienda, 1982, 1992, 2002 y 2017, Instituto Nacional de Estadísticas (INE). 
en el tramo de edad 15-64 años. Corresponde a la población económicamente activa (PEA) ${ }^{6}$, según las definiciones oficiales. Si en 1982 ese tramo de edad alcanzaba 59\%, en 1992 llegaba a 62\%, en 2002 a $66 \%$ y en 2017 a $72 \%$ de la población de la isla. Las proporciones en otros tramos de edad también manifiestan rasgos que importa comentar. En los censos comentados destaca la progresivamente menor proporción de niños y adolescentes hasta 14 años (de 38\% en 1982, a 34\% en 1992, a $30 \%$ en 2002, a $22 \%$ en 2017). En el otro extremo de los rangos etarios considerados, la proporción de adultos mayores se mantiene relativamente estable (3\%, 4\%, $4 \%$ y $6 \%$, respectivamente) Cf. Tabla 4. Sugerimos que la variación indicada en esas tres categorías de edad puede interpretarse mejor como el resultado del dinamismo de las migraciones recientes a Rapa Nui. Tanto el significativo crecimiento en términos absolutos de la población en Rapa Nui, como la particularidad de una cada vez más numerosa y porcentualmente dominante población en edad laboral, se origina en el aporte de fuerza de trabajo que por inmigración arriba desde el continente, resultante de la atracción económica que ejerce la expansión del turismo. Podría, todavía, advertirse una inflexión particular. La disminución de la proporción de población infantil en los períodos intercensales, mientras aumenta la población en edad económicamente activa y se mantiene una proporción regular de adultos mayores, sugiere que la inmigración, unida a condiciones demográficas locales (cambios en la edad de emparejamiento, la formación de hogares, el número y oportunidad de nacimiento de hijos, etc.) interviene en esta baja. De esto son parte los emparejamientos interétnicos y también entre inmigrantes. La última década, no obstante, pone de manifiesto incrementos de inmigración no asociadas primariamente a proyectos de radicación, sino a permanencias de trabajo estacional, anual y bianual en la economía del turismo.

\section{La ocupación Rapa Nui y no Rapa Nui en el turismo}

Según el Plan de Desarrollo de Isla de Pascua de 1972, utilizando datos de 1969, la ocupación de la población isleña se encontraba concentrada en las ocupaciones no calificadas (168 empleos). En segundo lugar, actividades por cuenta propia (68 ocupados) y mano de obra calificada (46 empleados). Queda clara, además, la prominencia de los servicios públicos como la principal fuente de empleos remunerados en ese momento. La fuerza de trabajo de ese entonces se contaba en 282 personas (ODEPLAN, 1972: 43-45). La mayoría de los isleños seguiría, en los hechos, ocupaciones combinadas. Las familias practicaban horticultura en parcelas, practicaban pesca, también poseían caballos y comenzaban a arrendar sus casas para el turismo. El sistema de ocupaciones era entonces bastante flexible. La agricultura en 1969 dispone de 117 parcelas dedicadas a esta actividad, sumado a la pesca y la construcción. La construcción de instalaciones hoteleras de HONSA y el aeropuerto ocupó por esos años a gran parte de la población, sin que dejaran sus ocupaciones originales. La producción primaria de agricultura y pesca ocupaba al $25 \%$ de la población empleada. El $6 \%$ se dedicaba a la artesanía y el 7,1\% actuaba en el sector privado, asociado a un desarrollo incipiente del turismo. En los años posteriores habría una importante pérdida de ocupación en la agricultura, correspondiendo con la expansión de la actividad turística (Porteous, 1981: 225). El perfil mixto y la relativa flexibilidad de la economía rapanui se ha mantenido, por lo menos

Tabla 4. Población total en Rapa Nui según períodos intercensal y rango etario

\begin{tabular}{|c|c|c|c|c|}
\hline \multirow{2}{*}{ Año } & \multirow{2}{*}{ Total, población } & \multicolumn{3}{|c|}{ Rango etario } \\
\hline & & $0-14$ & $15-64$ & $65 y+$ \\
\hline 1982 & 1.936 & $739(38 \%)$ & $1.150(59 \%)$ & $47(3 \%)$ \\
\hline 1992 & 2.764 & $949(34 \%)$ & $1.708(62 \%)$ & $107(4 \%)$ \\
\hline 2002 & 3.791 & $1.143(30 \%)$ & $2.499(66 \%)$ & $149(4 \%)$ \\
\hline 2017 & 7.750 & $1.675(22 \%)$ & $5.606(72 \%)$ & $469(6 \%)$ \\
\hline
\end{tabular}

Fuente: Elaboración propia con base en resultados censales 1982, 1992, 2002 y 2017.

Instituto Nacional de Estadísticas (INE). 
si se lo compara con las actividades y empleos no rapanui. No obstante, la combinación de trabajo asalariado, emprendimientos por cuenta propia, actividades de agricultura y pesca, actividades menores dependientes, e ingresos por rentas se relacionan y dependen crecientemente del turismo como núcleo aglutinador que organiza esa variedad y flexibilidad.

En las décadas siguientes al diagnóstico citado, la ocupación rapanui y no rapanui se transforma, destacando el incremento del trabajo asalariado y por cuenta propia no tradicional. En efecto, entre 1992 y 2002 el asalariamiento prácticamente se duplica $^{7}$; transformación que no es explicable por el empleo en las agencias estatales, que también crece, sino principalmente por la conformación de unidades de servicios para el turismo que amplifican relaciones contractuales sujetas a remuneraciones. Junto con ello, el trabajo por cuenta propia, especialmente el no tradicional (construcción, servicios al hogar, pequeño comercio), aumenta casi dos tercios durante el mismo período. Debido a que es a partir de la segunda mitad de la década de 1990 que se verifica un notable aumento del turismo en Rapa Nui, concuerdan entonces el período, los tipos generales y crecimiento hacia el que se vierte la ocupación en la isla. Sostenemos así que las ocupaciones han crecido y se han diversificado con la expansión del turismo, tanto para rapanui como no rapanui. De esta manera, el cambio en la estructura ocupacional isleña se verifica en el crecimiento y diversificación de los empleos; segundo, en un mayor asalariamiento y trabajo por cuenta propia no tradicional; tercero, en el incremento exponencial de la ocupación (el 2017 llega al 85,3\% de la población económicamente activa, con una PEA de $72 \%$ respecto de la población total (para un desglose cf. Tabla 5), su punto más alto en los últimos cuarenta años; y, por último, el crecimiento de la población económicamente activa y la ocupada se asocia directamente con la fuerza de atracción de trabajadores no rapanui desde la economía del turismo. A continuación mayores detalles de estos cambios.

La población residente de la isla prácticamente se cuadruplicó en 45 años. Pasa de 1936 personas en 1982 a 7750 en 2017, lo que habla de un crecimiento muy dinámico. A su vez, la población económicamente activa presenta un crecimiento igualmente sostenido y dinámico desde 1982 en adelante: de $59,4 \%$ en 1982 , a $61,8 \%$ en 1992 , a $65,9 \%$ en 2002 y $72,3 \%$ en 2017 respecto de la población total. Los ocupados respecto de la PEA, correlativamente, pasan de $54,6 \%$ en 1982 , a $66,1 \%$ en 1992 , a $73,3 \%$ en 2002 , a $85,3 \%$ en 2017 . $\mathrm{La}$ comparación con el país ayuda a dimensionar mejor el dinamismo señalado. En el 2017 en Chile la población económicamente activa era de $69 \%$ de la población y la ocupación cubría $45 \%$ de la PEA. La demanda de trabajadores asalariados y el incremento de oportunidades en el trabajo por cuenta propia serían los factores que inciden en la ampliación de la ocupación de la población no rapanui en la economía local y, con una distribución en categorías ocupacionales más diversa, en absorber el crecimiento de la población económicamente activa rapanui.

La Tabla 5 muestra una distribución ocupacional compartida entre rapanui y no rapanui, llegando a ser en 2017 una mayoría clara la de los ocupados no rapanui. Por lo demás, este año la población rapanui es una minoría tanto en la ocupación como en el

Tabla 5. Distribución de PEA, ocupación rapanui y no rapanui respecto de la población total

\begin{tabular}{llcrl}
\hline \multicolumn{1}{c}{ Categoría/año } & \multicolumn{1}{c}{1982} & 1992 & 2002 & 2017 \\
\hline Población censada & 1.936 & 2.764 & 3.791 & 7.750 \\
PEA & $1.150(59,4 \%)(\mathrm{a})$ & $1.708(61,8 \%)$ & $2.499(65,9 \%)$ & $5.606(72,3 \%)$ \\
Total ocupados & $628(54,6 \%)(\mathrm{b})$ & $1.129(66,1 \%)$ & $1.832(73,3 \%)$ & $4.782(85,3 \%)$ \\
Ocupados rapanui & - & $688(60,9 \%)(\mathrm{c})$ & $974(53,2 \%)$ & $1.844(38,6 \%)$ \\
Ocupados no rapanui & - & $441(39,1 \%)$ & $858(46,8 \%)$ & $2.938(61,4 \%)$ \\
\hline
\end{tabular}

\footnotetext{
a) Porcentaje respecto de la población total.

b) Porcentaje respecto de la PEA.

c) Porcentaje respecto de los ocupados.
}

Fuente: Elaboración propia a base de los datos del Censo de Población y Vivienda, 1982, 1992, 2002 y 2017. Instituto Nacional de Estadísticas (INE). 
total de la población global (44\% rapanui y $56 \%$ de no rapanui, según INE, 2017). En términos relativos ahora, la ocupación continental y de extranjeros sube rápidamente desde $39,1 \%$ en 1992 , a $46,8 \%$ en 2002 a 61,4\% en 2017. Esto es, se constituye en una mayoría de la población ocupada en la isla. La ocupación rapanui, a su vez, aumenta en términos absolutos, pero decrece en términos relativos $(60,9 \%$ en 1992, a 53,2\% en 2002, a 38,6\% en 2017); esto significa que el mercado de trabajo y ocupaciones absorbe de manera más dinámica a los no rapanui, especialmente en los nichos de trabajo dependiente que ofrece la economía del turismo. En suma, la ocupación de la población no rapanui se concentra hoy en el trabajo asalariado no especializado y cuenta propia menores, aunque su presencia se verifica en todas las categorías ocupacionales. Para el año 2017 casi dos tercios de la ocupación en Rapa Nui se encuentra cubierto por población no rapanui. Expresa un crecimiento sostenido de participación en el empleo desde 1992, en términos absolutos y porcentuales (cf. Tabla 5).

El conjunto de estos datos pone de manifiesto una realidad de pleno empleo y, a la vez, limitaciones locales para cubrir la demanda de trabajadores y oportunidades de trabajo existentes. Planteamos que en ese diferencial es donde se aloja la fuerza de atracción para los trabajadores no rapanui, en particular durante el período 2002-2017. Hipotetizamos que el crecimiento del turismo ha impulsado el surgimiento de empleos en un volumen tal que incentiva la llegada de mano de obra del continente para ocuparse en los crecientes empleos generados en los servicios turísticos, mismos que la disponibilidad de la mano de obra rapanui no alcanza a cubrir. Si esto es así, los no rapanui que han ido llegando a la isla, podemos sugerir, no "quitan trabajos" a los rapanuis; más bien se emplean en "cupos vacantes" y se ocupan desarrollando actividades por cuenta propia que se abren con el crecimiento de la demanda turística.

Acorde a lo ya presentado, los no rapanuis se ocupan principalmente en actividades asociadas al turismo, estos son los que tienen mayor presencia allí, numéricamente hablando. Mientras, los rapanuis se encuentran presentes en todas las ramas de la economía insular. Pero una descripción de la economía isleña del turismo basada en la ocupación es, no obstante, limitada. La propiedad de la tierra, de las instalaciones urbanas, viviendas y en general infraestructura donde se llevan a efecto las actividades relacionadas con el turismo recae en personas y familias rapanui. Por tanto, flujos importantes de recursos son captados por estos propietarios, lo que se traduce en una subeconomía rentista. Otro tanto ocurre con la propiedad y la dirección de las unidades económicas, en la gran mayoría de los casos en manos rapanui, lo que se expresa en la existencia de un segmento de pequeños empresarios, cuya capitalización e ingresos se diferencia claramente del resto.

Según la información provista por un censo de establecimientos relacionados directamente al turismo, realizado por los autores a fines de $2018^{8}$, el número de unidades encuestadas fue de $223 \mathrm{y}$, considerando rechazos y omisiones, llega a alrededor de 240 casos. Son importantes en número las unidades económicas integradas por una o dos personas ( 87 casos con $39,0 \%$ ), lo que las ubica en la definición de emprendimientos económicos personales o familiares; le sigue el grupo en que se desempeñan 3-5 personas (100 casos con 44,8\%). Una parte de esta categoría cabe en la definición de miniempresa si acaso incluye fuerza de trabajo asalariada. Ambos estratos representan poco más de cuatro quintos del total de establecimientos que prestan servicios al turismo $(83,8 \%)$. No destacan por la capitalización o un alto nivel de ingresos, sí en su capacidad de integración social con una base muy amplia de pequeñas unidades de trabajo, ingresos y prestación de servicios (alojamientos, confección y venta de artesanías y souvenirs, pequeños locales de comida, etc.). Considerando ambas categorías y su elevado porcentaje respecto del total, un sello distintivo de la economía del turismo en Rapa Nui radica en que está basada en economías familiares, una parte de estas integran fuerza de trabajo remunerada. Queda por establecer con suficiente detalle las características precisas de este empleo (roles principales, proveniente de la red familiar o externa a ella, estable o de tiempo parcial, formal e informal, composición solo monetaria de la remuneración o incluyendo otros aspectos como alimentación y alojamiento, etc.).

La siguiente categoría con 6 a 10 integrantes está representada por 36 unidades $(11,7 \%$ de los casos). Incorporan de manera regular fuerza de trabajo asalariada en cantidades variables según el tamaño y los rubros de servicios de que se ocupen. Pequeños hoteles, algunos turoperadores, restaurantes que sirven al turismo caen en esta categoría. Este grupo, unido a una parte de aquellas entidades con 
3-5 integrantes (que incorporan trabajo asalariado) constituye la miniempresa del turismo insular. Sumados los emprendimientos individuales, solo familiares y miniempresas (convencionalmente con 1-10 trabajadores regulares sujetos a salarios y formalización de sus actividades) el porcentaje de unidades llega a 95,5\% del total (cf. Tabla 6). De este modo, pequeñas unidades económicas unipersonales y bipersonales, anclados en $\mathrm{u}$ organizadas familiarmente, junto con otras que incorporan fuerza de trabajo de manera temporal o estable proveniente de la red familiar o externa (miniempresas), constituyen el núcleo socialmente extensivo de la economía del turismo en Rapa Nui.

Tabla 6. Número de trabajadores por establecimiento en la industria del turismo de Rapa Nui

\begin{tabular}{lcccc}
\hline $\begin{array}{c}\text { Trabaja- } \\
\text { dores }\end{array}$ & $\begin{array}{c}\mathrm{N}^{\mathbf{o}} \\
\text { estableci- } \\
\text { mientos }\end{array}$ & $\%$ & $\begin{array}{c}\mathrm{N}^{\mathbf{2}} \\
\text { personas }\end{array}$ & $\%$ \\
\hline $1-2$ & 87 & 39,0 & 136 & 12,5 \\
$3-5$ & 100 & 44,8 & 465 & 42,5 \\
$6-10$ & 26 & 11,7 & 209 & 19,1 \\
$11-20$ & 8 & 3,6 & 283 & 25,9 \\
21 y + & 2 & 0,9 & 1.093 & 100 \\
\hline \multicolumn{1}{c}{ Total } & 223 & 100 & 1.09 \\
\hline
\end{tabular}

Fuente: Elaboración propia con información de encuesta a establecimientos en la economía del turismo en Rapa Nui (nov.-dic. 2018).

De los restantes casos registrados $(4,5 \%$ del total) 9 califican por su tamaño, capitalización, volumen de empleo regular y niveles de ingreso como pequeñas empresas. La restante, por su escala de operaciones, se constituye en mediana empresa. Corresponden a las unidades de mayor tamaño en la economía del turismo de la isla. Según la composición son sociedades de capital con socios externos o entidades exclusivamente familiares. Varias de ellas forman parte de conjuntos de negocios de propiedad de familias rapanui, en que algunos prestan servicios al turismo y los demás forman parte de la economía de la isla más ampliamente considerada: supermercados, combustibles, transportes marítimos, servicios aeroportuarios, ferreterías y materiales de construcción, etc. Bajo estas consideraciones, agregando negocios en manos de los mismos propietarios e inversores, aumenta en número el grupo de medianas empresas ${ }^{9}$. Es notorio en estos casos su control por parte de familias con una larga trayectoria en la economía del turismo. En el área de comercio no relacionado directamente al turismo se verifica la presencia de algunas otras empresas en la categoría de pequeñas. Agregadas según mismos controladores pueden escalar a la condición de medianas.

La encuesta a establecimientos del turismo en Rapa Nui reportó 1093 personas que se desempeñaban en ellos cumpliendo distintos roles. Esta cifra se amplía si consideramos quienes trabajaban en Ma'u Henua, hasta alrededor de 1400 personas y todavía más durante el período anual de mayor afluencia de turismo (se estima en unas 300 personas extras). En los emprendimientos unipersonales y bipersonales se trata de autoempleo y alcanza a 136 personas $(12,4 \%)$ con $39 \%$ de las unidades económicas de servicios al turismo. Entre 3 y 5 integrantes, correspondientes a unidades familiares de autoempleo que incorporan otros miembros del hogar o contratados externos (incluyendo entonces el primer tramo de miniempresas) se agregan otras 465 personas $(42,5 \%)$ y $44,8 \%$ de las unidades económicas. El segundo tramo de las miniempresas con 6-10 integrantes abarca 36 casos $(11,7 \%)$ y congrega 209 personas $(19,1 \%)$. El resto corresponde a pequeñas empresas y al menos una mediana (10 casos y 4,5\%, con 283 personas que representan $25,9 \%$ de ocupaciones). Es posible agregar que la mayor variación anual en la incorporación de fuerza de trabajo corresponde a las pequeñas empresas y a la mediana. Las miniempresas que incluyen trabajadores externos, provenientes de la red familiar o de continentales, tienden a fidelizar en mayor grado sus dotaciones.

Según la misma encuesta, es mayoritario el empleo y autoempleo femenino con $73 \%$ de los casos, lo que imprime un sello de género particular a esta industria en la isla, al tiempo que hace presumir la presencia de estructuras de ingresos diversificadas, especialmente en el segmento de emprendimientos familiares uni y bipersonales y en la miniempresa. Considerando la pertenencia étnica, el empleo en el turismo es en gran medida continental (con $70 \%$ de los casos registrados), los extranjeros $9,1 \%$ (principalmente trabajadores haitianos, colombianos y peruanos) y los rapanui $20,9 \%$. De esta manera la ocupación en las actividades del turismo es principalmente femenina (algo más de dos tercios de ella según nuestros datos) y mayoritariamente continental (también con algo más de dos tercios de la ocupación y empleo). La 
diversificación de ingresos en estos actores es una apreciación válida, entonces, en la ocupación rapanui y en la continental relacionada a la población rapanui (normalmente aquella con mayor permanencia en la isla y con emparejamientos, hogares y familias). El resto depende básicamente de las remuneraciones que reciben.

Los continentales, de manera más definida que en las restantes categorías, se hacen presente en el trabajo dependiente. Esto es, aquellas personas que laboran y reciben remuneraciones de empleadores. La categoría de dependientes en servicios al turismo, comercio o administración alcanza así a $65 \%$ de las personas ocupadas en las actividades del rubro y está integrada principalmente por continentales. A su vez, en la de miniempresarios y emprendimientos uni, bipersonales, o que agregan algún(os) integrante(s) de la red familiar, corresponden a $23 \%$ de los ocupados, principal pero no exclusivamente rapanui. Otro grupo, todavía, es el de trabajadores en ocupaciones menores con $11 \%$ (jardineros, aseadores por tiempo parcial, personas que hacen reparaciones menores, etc.), donde es notoria la presencia de extranjeros. Por último, la categoría de empresario, gerente o administrador en una miniempresa del segundo tramo de tamaño (6-10 personas) o pequeña empresa alcanza a $1 \%$.

La industria del turismo de Rapa Nui genera un importante volumen de empleo directo, dependiente en servicios, comercio y administración. Un tercio de la ocupación es mini o microempresario y cuenta propia. Estos dos tipos de ocupación representan las categorías con mayor representación. El primero poblado en su mayoría por continentales, aunque también de extranjeros; el segundo por rapanui. Por lo demás, es principalmente femenino. Las posiciones en la ocupación de esta industria se organizan entonces siguiendo delimitaciones étnicas y prevalencia de género. En el capítulo siguiente abundamos en algunos aspectos de este tema.

\section{El control rapanui de la industria del turismo}

Luego de resolver la conectividad aérea en 1967 el interés turístico aumentó considerablemente, ahora con alcances globales. Para comienzos de la década de 1970 se podía decir que los rapanui mantenían una economía que se ligaba al aparato administrativo, a la industria del turismo y al comercio (Foerster y Montecino, 2017: 184). Para fines de los años de 1970 el turismo compite con la administración pública en su condición de principal economía monetarizada de la isla, y los servicios a visitantes se convierten en una alternativa concreta de generación de ingresos para la población local ${ }^{10}$. En 1975 gran parte de las residenciales y servicios turísticos son emprendimientos emergentes de personas y familias rapanui, aunque también se constata participación de continentales: Solamente 8 de los 25 pequeños negocios que operan en la isla son de continentales, lo que nos habla que la mayor parte de la actividad comercial está en manos rapanui, señalan Foerster y Montecino (2017:186-187). Según los archivos de la Gobernación Provincial de Isla de Pascua en 1975 hay 30 emprendimientos organizados con este objetivo: 4 agencias de viaje, el hotel "Hotu Matua" y 25 residenciales (P1590217-18-19, codificación propia). Además, la agrupación de residenciales y

Tabla 7. Prestadores de servicios turísticos registrados en Sernatur Isla de Pascua según tipo de actividad y condición de rapanui, continental o extranjero en el 2017

\begin{tabular}{lrrrr}
\hline \multicolumn{1}{c}{ Servicio } & Total prestadores & Rapanui & Continental & Extranjeros \\
\hline Alojamiento y hotelería & $157(59,5 \%)$ & 130 & 26 & 1 \\
Guía de turismo & $42(15,9 \%)$ & 32 & 5 & 5 \\
Artesanías & $6(2,3 \%)$ & 5 & 1 & - \\
Turismo aventura & $18(6,8 \%)$ & 15 & - & - \\
Servicios culturales & $4(1,5 \%)$ & 4 & - & - \\
Arriendo de vehículos & $2(0,8 \%)$ & 2 & 1 \\
Transporte al aeropuerto & $6(2,3 \%)$ & 15 & 4 \\
Agencias y turoperadores & $17(6,4 \%)$ & 8 & 4 & - \\
Restaurantes y similares & $12(4,5 \%)$ & $216(81,8 \%)$ & $40(15,2 \%)$ & $8(3,0 \%)$ \\
\hline Total & $264(100 \%)$ & & \\
\hline
\end{tabular}

Fuente: Elaboración propia con base en información del registro de prestadores de servicios al turismo de Sernatur, oficina de Rapa Nui (febrero 2017). 
la Junta de Vecinos ya por ese entonces manifiestan la necesidad de establecer límites y un control de parte de las autoridades para proteger el turismo como una actividad propia de la población isleña. Tempranamente, entonces, la división entre locales ${ }^{11}$ y continentales de más reciente aparición será activada como un argumento por posicionamiento y dominio en los servicios turísticos.

El registro de prestadores de servicio de la oficina de Sernatur en Isla de Pascua, ${ }^{12}$ complementa una visión de los servicios del rubro. Entendemos que un cierto volumen de unidades que operaban informalmente en 2016 no está registrado en estos cómputos. Los servicios son: prestadores de alojamientos y hotelería, guías turísticos, productores de artesanías, turismo aventura, servicios culturales, arriendo de vehículos, transporte de pasajeros al aeropuerto, turoperadores y restaurantes. Resumiendo, el $81,8 \%$ de los prestadores de servicio en el turismo son rapanui, el 15,2\% continentales y el $3 \%$ extranjeros, mostrando con esto la participación y prevalencia que las unidades rapanuis mantienen. La Tabla 7 muestra en detalle la participación rapanui, de continentales y extranjeros en los tipos de prestación de servicios al turismo.

La tabla precedente considera a quienes prestan servicios turísticos y sus controladores, activos y no activos. Por aquel entonces, esta repartición pública no ejercía supervisión de instalaciones y servicios ni entregaba certificación o acreditación, como se está haciendo ahora. Por otra parte, la formalización fiscal de las unidades económicas del turismo (su registro en Sernatur entre otros) no es completa, en particular con el uso de plataformas de reserva, transacción y prestación operadas mediante internet ${ }^{13}$. Por su parte, según la información de 223 unidades que obtuviéramos a fines de 2018, los alojamientos en sus distintas modalidades (hostales, residenciales, cabañas, lodge, hoteles, hotel boutique, etc.,) son el servicio al turismo más numeroso, con $46,2 \%$ de los casos. Le sigue el comercio al turismo con 30,0\%. Los servicios de alimentación orientados a turistas alcanzan el 14,3\%. Luego, turoperadores con 5,4\% y, por último, un grupo pequeño que incluye rent a car, transporte de personas, buceo y entrenamiento marino y otros con $4,1 \%$. Atendiendo a ambas fuentes (Sernatur e información propia), los alojamientos son el servicio al turismo con más unidades. A pesar de la diferencia en los esquemas de registro y de cambios que pudieran haberse producido desde
2016 (datos de Sernatur acumulados a esa fecha) y el encuestamiento censal de fines de 2018, el panorama general es convergente.

Una aproximación a los ingresos que obtienen estas unidades, según declaraciones propias y tomando como referencia el 2018, indica que $52 \%$ de los casos se posiciona en el rango 1 a 5 millones de pesos chilenos como ingresos mensuales brutos. Corresponde, principalmente, al rubro de alojamientos y locales de alimentación pequeños. Le siguen en número los casos de ingresos mensuales declarados entre 100 mil y 1 millón de pesos con el 34,5\%. Negocios pequeños de venta de artesanías en kioscos y galerías se encuentran aquí bien representados. El 6,3\% ocupa el rango de 5 a 10 millones y corresponden a pequeños hoteles, restaurantes, y turoperadores con operación limitada. El 3,6\% entre 10 a 20 millones (hoteles y turoperadores); $1,8 \%$ de 20 a 50 millones y por último el $0,9 \%$ con 50 y más millones, que lo alcanzan las pequeñas empresas mejor posicionadas y una mediana.

Antecedente de importancia, según el mismo estudio, es que la mayor parte de las unidades de servicios al turismo son negocios familiares; esto es, creados, organizados y dirigidos por los integrantes de un hogar y mantenidos en esa condición, independientemente de la incorporación de fuerza de trabajo externo o no, la escala relativa de las operaciones y capitalización o los alcances en la formalización de sus actividades. El 89,2\% de los casos encuestados caen en este grupo. El resto corresponde a sociedades comerciales con $10,8 \%$; esto es, entidades con aportes de capital o activos para la conformación de una empresa de servicios con más de uno o varios socios. En algunos casos conocidos se trata de socios continentales que entregan financiamiento. Corresponden principalmente a pequeñas empresas y una mediana. Lo anterior refuerza la definición del carácter mayormente familiar de la industria del turismo en Rapa Nui.

Asimismo, todas las unidades de servicios, con alguna excepción como el Hotel Hanga Roa, se emplazan en dependencias, casas o terrenos de propiedad rapanui ${ }^{14}$. La condición de tenencia del lugar e instalaciones en que o desde las que se prestan los servicios indicados es heterogénea: propiedad particular, préstamos, arriendo, cesiones y comodatos. Tenemos entonces una mayoría de unidades de prestación de servicios turísticos de personas y familias rapanui o que integran rapanui 
y continentales, en terrenos, sitios, dependencias e instalaciones de propiedad rapanui $(81,8 \%$ según la Tabla 7). Y una minoría (18,2\% sumando continentales y extranjeros) que mantiene estas unidades de servicio, pero en terrenos e instalaciones cuya propiedad es rapanui y a la que acceden mediante arreglos económicos en que dominan rentas de arriendo.

Factores internos y externos de distinta índole se conjugan en una agencia económica rapanui exitosa. La posición privilegiada de dominio de la tierra, unido a las limitaciones para que el Estado entregara tierras fiscales a otros particulares, posibilitó conformar en terrenos rapanui servicios de turismo como alojamiento y hotelería, agencias y turoperadores, restaurantes y similares, en la década de los años setenta y ochenta, en plena emergencia de esta economía. La inversión continental en el turismo de la isla, en asociación con rapanuis, se materializó más tarde, cuando a finales de la década de 1990 se la visualiza como un destino global para un turismo de intereses especiales. De esa tipificación son responsables en buena medida las campañas de agencias estatales en el extranjero que buscaron posicionar el Desierto de Atacama, Patagonia e Isla de Pascua como destinos turísticos particulares. La afluencia masiva de continentales que permanecen como residentes en la década de 2000 también supuso interés por conformar unidades de servicios turísticos, ahora desde capacidades económicas más limitadas. Como señalamos más arriba, tempranamente se registran acciones tendientes a contener y limitar el acceso de población no rapanui a la prestación de servicios turísticos, lo que se ha intensificado en la última década; aunque por otra parte es en terrenos, viviendas e instalaciones rapanui que la hoy numerosa actividad por cuenta propia de continentales tiene lugar (por ejemplo el comercio de abastos), y ello supone rentas que interesan a esos propietarios particulares rapanui.

Mientras en el nivel de los individuos, hogares y unidades familiares rapanui se aprecia fluidez de relaciones, ajustes y arreglos con continentales para el desarrollo de sus emprendimientos y trabajos en el área del turismo y relacionados, en el nivel gremial local se ha mantenido una consistente posición de rechazo y acciones de contención tendientes a impedir o, al menos, limitar esa participación. Y, a la vez, mantener y reforzar la posición de dominio y control en la industria. La acción de la Cámara de Turismo creada en 1998, una iniciativa de empresarios con composición fundamentalmente rapanui, ha actuado en el sentido indicado, a la vez que acompañado y apoyado el desarrollo de sus asociados. La integran los principales hoteles y prestadores de servicios turísticos.

Es importante mencionar otros antecedentes. La Ley Indígena 19.253 entrega un marco de reconocimiento y acción afirmativa para que desde la institución generada (la Corporación Nacional de Desarrollo Indígena, Conadi) y otros organismos del Estado (mediante convenios interagencias, por ejemplo) intervengan en favor del turismo local. Esto se materializa por medio del organismo estatal, derivado de la ley anterior, la Comisión de Desarrollo de Isla de Pascua (Codeipa) con participación rapanui. En esta misma comisión tiene presencia la autoridad comunal, siempre rapanui en las últimas décadas, con algunas atribuciones en la regulación de las actividades del turismo. La acción del Servicio Nacional de Turismo (Sernatur) también tiene incidencia en labores de promoción, información y articulación con otras agencias del Estado y cadenas nacionales y globales de servicios, cuestión que habría sido regularmente mejor aprovechada por el grupo de empresarios locales una vez que este estamento se fue formando.

Varias son las fuentes de financiamiento que permitieron el rápido crecimiento de una oferta de servicios turísticos en Rapa Nui (en 1975 son 30 y 264 entidades prestadoras en 2017; 223 informadas a fines de 2018 según nuestro propio estudio, sin considerar guías de turismo) y la formación de un estrato diferenciado de empresarios locales. Primero, los más numerosos, los autoemprendimientos pequeños, familiares, que se van conformando progresivamente con ahorros propios, rentas y otros ingresos, a lo que se suma en su momento ingresos obtenidos luego de su puesta en marcha; es el caso de la mayoría de los hospedajes pequeños existentes en la isla. Es posible que algún subsidio estatal se haya obtenido en su transcurso o en relación con ampliaciones y mejoras (destacan en este punto transferencias desde Conadi). El segmento mejor situado en la economía de los servicios turísticos pudo haber arrancado en los albores de esta actividad como pequeño emprendedor, pero el escalamiento a la condición de miniempresario o pequeño, supuso incorporaciones de capital proveniente de la asociación con inversionistas externos, continentales o extranjeros, el acceso a préstamos subsidiados o directamente subsidios estatales para inversión 
(por ejemplo, la Conadi), por medio de la banca privada cuando ello fue posible y conveniente; o una combinación de las anteriores.

Un nexo fundamental es el que conecta turoperadores nacionales y globales con operadores locales, hoteles pequeños y medianos. Ello garantiza, sinérgicamente, un flujo de pasajeros sostenido, ingresos importantes, acumulación y reinversión, compra de tierras, nuevos vínculos externos y servicios, etc. Algunos empresarios rapanui gustan destacar su desarrollo económico como el resultado del trabajo sostenido y la habilidad personal para impulsar ese crecimiento. Estas ideas no hacen justicia a las condiciones y manejo de oportunidades que hicieron fructificar esa dedicación personal, y tampoco al hecho que a partir de cierto momento de crecimiento esa unidad económica se sostiene en el trabajo dependiente.

La expansión del turismo demandó un amplio contingente de trabajadores en la construcción y en los servicios asociados, lo que indujo una transformación demográfica visible, mediante población continental que llegó a trabajar y residir en la isla. Ya en 2002 los continentales tienen una envergadura cercana a la mitad del total de la población de la isla. Es una incorporación heterogénea, en cuanto a la distribución de las posiciones sociales y económicas. Una parte de ellos encuentra condiciones para hacerlo autónomamente (trabajo por cuenta propia, emprendimientos en comercio y transporte, servicios de alimentación, etc.). Otro segmento se incorpora como dependiente en establecimientos mayoritariamente orientados a los servicios turísticos. Un número creciente de ellos es trabajador temporal, estacional o por períodos más largos, sujeto a acuerdos y contratos precarios. En conclusión, por tratarse principalmente de trabajadores, prestadores de servicios y cuenta propia menores, los continentales ocupan por lo general posiciones bajas en la estructura económica de la industria.

En suma, los continentales tienen una participación laboral y de prestaciones de servicios numerosa, pero no de predominio ni en volumen ni en dominio en el funcionamiento de la industria turística. Se encuentran, por tanto, en una posición relativa de inferioridad en comparación con los actores económicos rapanui. Al interior de estos, a su vez, se advierte una notoria estratificación socioeconómica. Al respecto, el trabajo continental dependiente ayuda a reproducir esa posición de dominio y control rapanui, a la vez que la propia diferenciación económica interna en la economía del turismo y, por extensión, en la sociedad insular.

Douglas Porteous advirtió en 1981 una incipiente competencia por los recursos del turismo y declara que: un sistema de turismo organizado por los continentales limitaría las oportunidades de los isleños para controlar su modo de vida y podrían haber desaparecido (Porteous, 1981: 238). Ninguno de estos temores se ha hecho realidad, antes al contrario. Pero es cierto que la presencia masiva de continentales y su activa participación en el turismo levanta la pregunta por la posición rapanui allí, genera preocupaciones por el futuro de su isla y respecto de la principal industria que dinamiza su economía, catapulta posiciones de oposición y controversia, y estimula el paso a la acción colectiva. Su primera manifestación, suficientemente expresiva, fue la toma del Aeropuerto Mataveri ${ }^{15}$. Un punto de llegada, la negociación de un proyecto de "ley de residencia" para regular la crisis demográfica y ambiental en curso $^{16}$.

\section{Conclusiones}

Las transformaciones modernizadoras y la economía del turismo cambiaron profundamente muchas dimensiones de la vida en Rapa Nui a partir de la segunda mitad de la década de 1960. Efectos primarios en la vida social aquí informados se verifican en dimensiones demográficas, ocupacionales y económicas. En primer lugar, la población residente crece muy rápidamente, cuadruplicándose en un lapso de poco más de cuatro décadas (de 1936 habitantes en 1982 a 7750 en 2017), producto del crecimiento interno, inmigración rapanui de retorno y, en especial, inmigración de continentales y extranjeros que establecen residencia en la isla. Ese crecimiento acelerado se explica por la atracción que ejerce el rápido crecimiento del turismo, conjunto de actividades expansivo en empleos y ocupaciones (construcción y mantenimiento, hotelería y alojamientos, servicios de transporte y traslado, comercio de abastos, otros servicios, etc.). El trabajo por cuenta propia en oficios y microemprendimientos de alojamiento, alimentación y comercio, el empleo dependiente asalariado en hospedajes, hoteles o restaurantes, además de rentas de bienes raíces, etc., es la fuente principal hoy de ingresos de la población, seguida de cerca por la administración del Estado y servicios conexos. La 
oferta de empleo y posibilidades de ingreso por cuenta propia es muy dinámica, tanto que en las últimas décadas se asiste a una situación de virtual empleo pleno (alto porcentaje de PEA producto de la inmigración acelerada, pero también sostenido crecimiento de la proporción de ocupados superando ampliamente el promedio nacional).

En la estructura social se establece una configuración paradojal. Por una parte, las libertades de residencia y circulación, unido al aumento de visitantes y de residentes continentales que laboran en la isla generó, primero, una permanente e intensa interacción social de rapanui con personas de otros orígenes y a la inversa; y, segundo, relaciones y lazos sociales de alianza y cosanguinidad en el ámbito del parentesco, pero también vínculos de vecindad en los espacios residenciales, de amistad, de convivencia y convivialidad en los medios escolares y laborales, asociatividad en actividades económicas, así como relación de clientes en los servicios turísticos. Y en ello personas y familias rapanui han sido muy activas tanto en la isla como en Chile continental y en el extranjero. Con todo esto tiene lugar una profusa integración social. Pero, por otra parte y en contraste con lo anterior, en esta nueva configuración persisten definiciones y posicionamientos de identidad y diferencia entre "rapanuis" y "contis". La puesta en funciones de una política multicultural y afirmativa en la década de 1990 confirma y desarrolla las diferencias señaladas, ahora desde un marco de legitimidad provisto por el cambio, limitado y todo, de un paradigma de integración por asimilación, a otro multiculturalista que supone garantías y derechos de minoría étnica. Y todavía más, la masiva participación de continentales en la economía isleña, muy importante ya en la década de 2000, confronta a rapanui y "conti" con la masividad de esta presencia residente, la presión por recursos y servicios, la competencia en ciertos nichos laborales e incluso competencia en algunos servicios al turismo. Lo anterior no obstante que el crecimiento acelerado del empleo resultante de la expansión del turismo tiene a los continentales y extranjeros, con una mayoría de mujeres, como principales proveedores de mano de obra, en servicios que empresarios y emprendedores por cuenta propia rapanui sostienen una posición dominante.

Su consecuencia general es una diferenciación interna por desigualdad económica y de poder en la población rapanui, que se manifiesta con claridad en la estratificación social existente (categorías socioeconómicas y de poder) y, al mismo tiempo, entre categorías étnicas ("rapanuis" por una parte, y "contis, "tires" y "extranjeros" por otra). Estos últimos ocupan en la estratificación social posiciones como trabajadores asalariados, trabajadores por cuenta propia en oficios menores y mini o microemprendimientos. Los primeros son una contundente mayoría en las posiciones de propietarios, gerentes y administradores de hoteles y otros servicios de turismo o relacionados a esta industria; otro tanto con las mini y microunidades mejor capitalizadas. El segmento rapanui en el trabajo asalariado y dependiente es visible, pero ha ido disminuyendo en el tiempo, en favor del trabajo remunerado ocupado por continentales. Esta configuración de la estratificación económica es nueva en Rapa Nui. También en nuevos términos se reproduce la diferenciación basada en distinciones, delimitaciones y fronteras de etnicidad ("rapanui" - "conti"). Ambas se refuerzan recíprocamente. Posicionamientos rapanui en organizaciones como la Cámara de Turismo, en la defensa de la tierra, el control político municipal, la participación en comisiones e instituciones como Codeipa, el reciente control del Parque Nacional Rapa Nui por la organización Ma'u Henua, la existencia de grupos de presión según facciones y grupos parentales, e incluso la animosidad a flor de piel contra los "tire" (continentales de segunda categoría) actúan en favor de producir y reproducir la posición rapanui dominante.

Pero esas posiciones económicas actúan en el sentido de producir, reproducir y ampliar la diferenciación socioeconómica creada en este proceso. Estratificación que después de establecida está empezando a ser materia de controversia interna. Esta es una primera paradoja: las oportunidades abiertas por la modernización en Rapa Nui, de lo que forma parte una consistente defensa y promoción de intereses en tanto grupo étnico, tiene como una de sus consecuencias una marcada diferenciación económica y de poder interna. Una segunda paradoja se presenta ahora en las relaciones interétnicas: el dominio rapanui de las posiciones de influencia, representación y poder local, unido al dominio que ejercen en una economía del turismo en expansión, hace de la población inmigrante una categoría funcional a ese dominio rapanui, a la diferenciación económica en curso y a su profundización. Si en décadas pasadas se advirtió a los "conti" competidores potenciales en la nueva economía emergente, controlada esa 
amenaza el rápido aumento de población continental y extranjera asalariada en la isla hace posible la expansión actual de esa economía. Los atributos de la economía de Rapa Nui y la etnicidad están entonces relacionados, como lo está la distribución ocupacional y la estratificación socioeconómica y social. En todas estas dimensiones la economía del turismo tiene hoy, con un paréntesis en la presente coyuntura de pandemia, un papel decisivo como la esfera en la que se producen y reproducen posiciones ocupacionales, de ingresos, la diferenciación económica y con ello la particular configuración de estratificación social y etnicidad existente.

\section{Agradecimientos}

Este trabajo forma parte del Proyecto Fondecyt Regular No 1150573, dirigido por el autor principal.
Como parte de ello, una investigación doctoral en el Programa de Doctorado en Antropología UCNUTA (Instituto de Arqueología y Antropología (IAA), Universidad Católica del Norte), institución a quien agradecemos la oportunidad de su desarrollo. Agradecemos también a Fondecyt y a Conicyt, la provisión de recursos empleados en esta investigación.

En Rapa Nui compromete nuestro agradecimiento la Gobernación de Isla de Pascua, la oficina Provincial de Sernatur y la Comunidad Indígena Ma'u Henua. También el Centro de Investigaciones Interculturales e Indígenas, CIIR, Universidad Católica de Chile. La aplicación del cuestionario con el que se obtuvo algunos de los datos aquí presentados fue posible gracias al Departamento de Comunicaciones Ma'u Henua. A ellos expresamos todo nuestro agradecimiento por su apoyo.

\section{Referencias Citadas}

Arancibia, V.

2009 Rapa Nui: iorana te ma'ohi. Dilemas estratégicos. Ediciones Universidad Católica de Chile, Santiago. Arthur, J.

2015 Reclaiming Mana: Repatriation in Rapa Nui. Tesis doctoral. Universidad de California, Los Ángeles.

Concha, R.

2017 Dinámica del turismo y transformaciones en los usos del patrimonio en Isla de Pascua. Tesis de doctorado en Gestión de la cultura y el patrimonio. Universidad de Barcelona.

Delsing, R.

2017 Articulando Rapa Nui. Políticas culturales polinésicas frente al Estado chileno. LOM Ediciones. Santiago.

Faba, P.

2012 Entre le continent et l'île. Dimensions de la patrimonialisation de Rapa Nui (île de Pâques), Chili. Tesis de doctorado en Historia del Arte. Université Paris I, PanthéonSorbonne, París Francia.

Fajreldin, V.

2017 Colección cultura y patrimonio inmaterial de Rapa Nui. Tres volúmenes, Ediciones Ocho libros, Santiago.

Fischer, Steven

2005 Island and the End of the World. The turbulent History of Easter Island. Reaktion Books.

Foerster R. y Montecino S.

2018 "La lucha de los rapanui por agenciar el turismo en la Isla (1946-1980)". Cuadernos de Historia. Número 49. Universidad de Chile.

Foerster R. y Montecino S.

2017 "Unidad Popular y Golpe Militar en Rapa Nui. Informes de la Gobernación Junio y Octubre 1973”. Cuadernos de Historia. Número 47. Universidad de Chile.

Foerster, R., Ramírez, J. Moreno Pakarati, C.

2014 Cartografía y Conflicto en Rapa Nui. Rapanui Press, Santiago.
Muñoz, D.

2017 Diaspora rapanui (1871-2015) L'île de Pâques, le Chili continental et la Polynésie française. Une ethnographie historique de la mobilité dans une société transnationale. Tesis de Doctorado en Antropología Social y Ethnología, EHESS-Marseille, Francia

McCall, G.

1975 "Shorter Communications. Sympathy and Antipathy in Easter Islander Chilean relations". The Journal of the Polinesian Society. 84, pp. 476-475.

McCall, G.

1976 Reaction to disaster: Continuity and Change in Rapanui Social Organization, Canberra, Australian National University.

Oficina de Planificación Nacional (ODEPLAN)

1972 Plan de desarrollo de Isla de Pascua.

Porteous, D.

1981 The Modernization of Easter Island. Western Geographical Series. Universidad of Victoria. Canadá.

Rojas R. y Gundermann H.

2020 "Modernización, economía del turismo e interetnicidad en Rapa Nui (1966-2017)". Revista Austral de Ciencias Sociales 39: 169-192, 2020 DOI: 10.4206/rev.austral.cienc. soc.2020.n39-09

Thompson, W.

1891 En From the Report of the National Museum, 18881889, pp. 447-552. Publicado con el nombre: Te Pito Te Henua, or Easter Island, by William J. Thompson.

Documentos consultados

Censo de Población y Vivienda

1982 Bases de datos. Instituto Nacional de Estadísticas.

Censo de Población y Vivienda

1992 Bases de datos. Instituto Nacional de Estadísticas. 
Censo de Población y Vivienda

2002 Bases de datos. Instituto Nacional de Estadísticas.

Censo de Población y Vivienda

2017 Bases de datos (Consulta en línea http://www.ine.cl). Instituto Nacional de Estadísticas.

\section{SERNATUR}

2018 Bases de datos. Alojamientos, tour operadores, Guías de turismo.
SERNATUR.

1978-2010 Archivo histórico oficina provincial Isla de Pascua. SERNATUR.

2002-2013 Informe Anual de Turismo.

SERNATUR

1979 Isla De Pascua Plan Operativo. Versión preliminar.

\section{Notas}

1 Definición alineada con el glosario de la Organización Mundial del Turismo (OMT) http: // www2unwto.org.

2 Fuente: Informe Anual de Turismo, años 2002-2013 (Instituto Nacional de Estadísticas, INE y Servicio Nacional de Turismo Sernatur). La metodología empleada consiste en declaraciones de ocupación de camas, solicitadas a los establecimientos, usualmente mediante llamados telefónicos. Las posibilidades de subdeclaración son notorias. Además, no está disponible, hasta donde sabemos, el registro de pasajeros de la línea aérea (Lan Chile, ahora Latam) que traslada visitantes desde el continente americano (Santiago, por períodos también Lima) y desde Polinesia (Tahiti). Tampoco se dispone de registros de visitantes desembarcados desde cruceros de turismo que recalan en la isla y que durante algunas horas recorren el área urbana y, algunos, se dan tiempo para visitar el Parque Nacional Rapa Nui.

3 Los visitantes efectivos en el período pudieran ser algunos centenares menos, debido a la práctica de hoteleros y turoperadores importantes de comprar por anticipado boleto para sus clientes. Asimismo, no conocemos la existencia de uso de un boleto por más de una persona, dividiendo los puntos de visitas. Excepciones como las indicadas no alteran el orden de magnitud, envergadura o volumen global de la afluencia de visitantes a la isla en el presente.

4 Información de interés se encuentra también en Arancibia et al. 2009; la relación entre cultura, patrimonio y turismo es abordada por Faba, 2012; Arthur, 2015; Concha, 2017; Delsing, 2017, y por la compilación de Fajreldin, 2017. El nexo entre mercado y cultura en Rapa Nui es materia, por derecho propio, de una discusión que lo aborde con la extensión debida. Ello no es posible en el marco de un artículo cuyo objeto es distinto.

5 La elaboración de esta tabla se realizó con la información de los censos de población y vivienda de los años 1982, 1992 y 2002, del Instituto Nacional de Estadísticas (INE). No se utilizó el censo 2012 por recomendación del propio INE debido a que contiene graves errores de ejecución. Fue desechado por dicha institución en espera del censo de corrección finalmente realizado en 2017.

6 La población económicamente activa en Chile (PEA) según el Instituto Nacional de Estadísticas (INE) es la población entre 15 y 64 años de edad que esté ocupada, cesante o buscando trabajo por primera vez.

7 Información de categorías ocupacionales están disponibles en los Censos de Población y Vivienda de 1992 y 2002. En el censo abreviado del 2017 no se consideró esta dimensión, haciendo imposible realizar un cotejo directo seguro.
Información obtenida en entrevista con Sergio Rodríguez, Instituto Nacional de Estadísticas (INE).

8 La información proviene de una encuesta censal realizada en el marco de la investigación del doctorante Sr. Roberto Rojas y del Proyecto Fondecyt Regular $N^{\circ} 1150573$, bajo la supervisión y dirección del Dr. Hans Gundermann (Doctorado en Antropología UCN-UTA). Se contó también con el valioso apoyo institucional y material de la Comunidad Indígena Polinésica Mau Henu'a con quien se mantiene un convenio de colaboración recíproca. La encuesta censal consideró un cuestionario con datos del establecimiento proporcionados, preferentemente, por el o los propietarios, o quien ejercía la dirección o administración del mismo. El segundo cuestionario recogió información individual a una persona que se desempeñaba en el establecimiento, elegida al azar entre quienes lo integraran. Esto último no siempre se consiguió por razones de composición (establecimientos unipersonales), de oportunidad (se pudo reiterar solo una vez las visitas), o autoridad (solicitud de los requirentes por encuestar a determinada persona y no otras), por lo que en este último caso el cuestionario recogió información valiosa de categorías de sujetos y no de una muestra al azar. La encuesta se realizó en noviembre y diciembre de 2018 a 223 establecimientos, cuyos rubros estaban directamente relacionados con el turismo: alojamientos, hoteles, turoperadores, comercio al turismo, restaurantes de turismo y establecimientos de recreación. Hubo 15 rechazos, lo que lleva a 238 establecimientos encuestados activos en ese momento. Posteriormente verificamos un par de casos más que la búsqueda de ese momento omitió. El universo de establecimientos activos en esos meses intermedios (el período de alta afluencia y mayor activación de la economía del turismo es enero y febrero) fue entonces de 240 unidades aproximadamente. Registros municipales y del Servicio Nacional de Turismo (Sernatur) son acumulativos y por tanto más abultados, eventualmente parciales por informalidad, por lo que optamos por la identificación basada en el conocimiento local experto y revisión calle por calle y sector por sector de Moeroa, Hanga Roa, Mataveri y Tahai.

9 No se considera en estos cómputos la Comunidad Indígena Polinésica Ma'u Henua, administradora del Parque Nacional Rapa Nui, con aproximadamente 300 contratos para, principalmente, trabajadores rapanui. Por condiciones particulares a la configuración económica y del empleo en Rapa Nui una proporción alta de los contratados era fuerza de trabajo disponible. Su contratación no impactó significativamente, entonces, la ocupación existente. 
10 Los servicios de alojamiento, alimentación, asistencia y transporte al interior de la isla tuvieron desde el inicio participación rapanui. Para absorber la creciente actividad turística se requirió también acceso y creación de "comodidades" (mobiliario, enseres del hogar, televisión, más recientemente señal de internet), mejor provisión alimentaria y puesta a punto del transporte local.

11 Todavía el etnónimo "rapanui" no se ocuparía como un elemento discursivo central en un contexto de este tipo, sino que se aludiría a mejores derechos basados en el dominio histórico y la residencia continua.

12 Esta información obtenida el día seis de febrero de 2017 mediante el servicio de transparencia chilegob.cl.

13 La excepción tributaria que aplica a Rapa Nui habría llevado a maniobras de personas y empresas externas para registrar domicilio, nominalmente, en la isla buscando ventajas impositivas. El registro que mantiene el Servicio de Impuestos Internos (SII) al 2016 llega a 153 empresas, de estas 90 son de intermediación financiera y 63 de otros rubros. De las 90 empresas de intermediación financiera solo 5 operarían realmente en la isla.
14 Para profundizar en el tema de propiedad, traspaso y control de la tierra revisar Foerster, Ramírez y Moreno Pakarati, 2014.

15 Por ejemplo, el 15 y 16 de agosto del 2009 se produjo la toma del aeropuerto Mataveri poniendo de manifiesto la demanda por regulación de la circulación de la población continental por parte de la población isleña, en específico la población continental en Rapa Nui. El 18 agosto del 2009, el periódico La Tercera titulaba: “Una veintena de habitantes de Isla de Pascua quiso hacer pública su demanda como Parlamento de Rapa Nui y se tomó el aeropuerto de Mataveri el fin de semana, lo que ocasionó la suspensión de los vuelos a ese territorio insular".

16 Su resultado concreto actual es la Ley No 21.070. Queda fuera de los objetivos de este artículo el análisis del movimiento de oposición a la inmigración continental y extranjera, sus claroscuros, los efectos también controversiales de la aplicación de la Ley № 21.070, así como la crisis actual generada por el Covid 19, y cómo ello pueda modificar la diferenciación social y étnica desarrollada con base en la economía del turismo. 